\title{
Higher education delays and shortens cognitive impairment. A multistate life table analysis of the US Health and Retirement Study
}

\author{
Mieke Reuser • Frans J. Willekens • \\ Luc Bonneux
}

Received: 5 August 2010/Accepted: 3 February 2011/Published online: 20 February 2011

(C) The Author(s) 2011. This article is published with open access at Springerlink.com

\begin{abstract}
Improved health may extend or shorten the duration of cognitive impairment by postponing incidence or death. We assess the duration of cognitive impairment in the US Health and Retirement Study (1992-2004) by self reported BMI, smoking and levels of education in men and women and three ethnic groups. We define multistate life tables by the transition rates to cognitive impairment, recovery and death and estimate Cox proportional hazard ratios for the studied determinants. 95\% confidence intervals are obtained by bootstrapping. 55 year old white men and women expect to live 25.4 and 30.0 years, of which 1.7 [95\% confidence intervals $1.5 ; 1.9]$ years and $2.7[2.4 ; 2.9]$ years with cognitive impairment. Both black men and women live $3.7[2.9 ; 4.5]$ years longer with cognitive impairment than whites, Hispanic men and women 3.2 [1.9; 4.6] and 5.8 [4.2; 7.5] years. BMI makes no difference. Smoking decreases the duration of cognitive impairment with $0.8[0.4 ; 1.3]$ years by high mortality. Highly educated men and women live longer, but 1.6 years $[1.1 ; 2.2]$ and 1.9 years $[1.6 ; 2.6]$ shorter with cognitive impairment than lowly educated men and women. The effect of education is more pronounced among ethnic minorities. Higher life expectancy goes together with a longer period of cognitive impairment, but not for higher levels of education: that
\end{abstract}

M. Reuser · F. J. Willekens · L. Bonneux ( $\square)$

Netherlands Interdisciplinary Demographic Institute (NIDI), P.O. Box 11650, 2502 AR The Hague, The Netherlands e-mail: bonneux@nidi.nl

M. Reuser · F. J. Willekens

University of Groningen, Groningen,

The Netherlands extends life in good cognitive health but shortens the period of cognitive impairment. The increased duration of cognitive impairment in minority ethnic groups needs further study, also in Europe.

Keywords Cognitive impairment - Multistate life tables . Body mass index $\cdot$ Smoking - Educational status .

Ethnic groups

\section{Introduction}

Cognitive impairment is a major cause of dementia and associated disability and care dependence in aging societies. The age specific prevalence of dementia doubles with every 5 years of age, from approximately $1.5 \%$ in persons aged 60-69 to $40 \%$ among nonagenarians $[1,2]$. In the absence of effective interventions, numbers of people with dementia will increase as a simple consequence of an increase in the size of the elderly population [3]. This implies large increases of long term care needs. We used multistate life tables (MSLT) to identify duration of cognitive impairment in the synthetic (imaginary) cohort of a life table (see Fig. 1). MSLT is an important demographic tool to document life course processes, here the process to cognitive impairment and death [4]. Contrary to the classic (Sullivan) healthy life table [5], which combines prevalence with event rates, the MSLT is internally consistent by building exclusively on event rates [6]. The results of MSLT are highly intuitive by translating age specific event rates in measures of duration in the life table: how long individuals at a certain age will live in a specified good and bad health condition. Conditioned by risk status, the results assist both individuals and policy makers in health planning. 


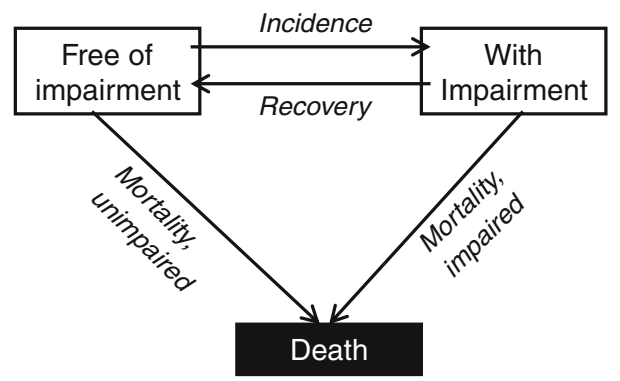

Fig. 1 Structure of a multi state life table model with two health states. The transitions are age dependent and conditioned by risk status

Old age and genetic susceptibility are firmly determined as causes of dementia. In European population, incidence rates of dementia among women are higher, often attributed to mortality selection among men [7]. Current evidence supports an important role for vascular risk factors in the clinical manifestation of cognitive decline [2, 8-10]. Blood pressure is correlated to dementia, but the relation depends on the life course $[11,12]$. Obesity shows the same life course dependency. A higher BMI in mid life has been related to an increased risk of dementia, mediated by vascular risk. But later in life, an accelerated decline of BMI predicts Alzheimer's disease [13]. Elderly smokers have increased risks of dementia and cognitive decline [14], but the role of smoking in shortening the life span is indisputable-which might shorten the life span with cognitive impairment at the end of life.

There is sufficient evidence that psychosocial factors such as educational attainment, social networking and mentally stimulating activities protect against dementia [2]. Last but not least, there is a clear association of incidence and duration of dementia with minority ethnic groups, observed in several countries and several ethnic minorities [15-19]. The reasons why minority ethnic groups suffer more and longer from dementia are ill understood but certainly manifold, partly linked to lower education and social deprivation [20].

The aim of this paper is to support future epidemiologic-demographic predictions of disability by assessing the duration of cognitive impairment and the effect of risk factors on this duration. We used data from the publicly available HRS study, a model for the European SHARE study (http://www.share-project.org/). The HRS had then seven rounds of interviews, SHARE but the first round. In the HRS study, cognitive impairment was defined by the Telephone Interview Cognitive Screen (TICS) or proxy-interviews [21].. We used MSLT to assess the duration of cognitive impairment, dependent on age, sex, ethnic status and the risk factors BMI, smoking and education.

\section{Methods}

Data and study population

We used the RAND version F of the Health and Retirement Study (HRS) data file containing the HRS and the Asset and Health Dynamics Among the Oldest Old (AHEAD) which began in 1992 and 1993, respectively, and were merged in 1998. The Children of the Depression cohort and War Baby cohort were included. We will refer to the total survey as HRS. The HRS survey includes a nationally representative sample of initially non-institutionalized individuals, and spouses, who were re-interviewed biannually, with oversampling of minority ethnic groups. We used data from 7 waves from 1992 to 2004. Response was on average $86 \%$. Data on vital status and month and year of death are obtained through the mortality register (the National Death Index) and exit interviews.

Cognitive impairment in the AHEAD93 and HRS98 was measured by a modified version of the TICS (The Telephone Interview Cognitive Screen) instrument, a telephone interview adapted from the MMSE (mini mental state examination) [21, 22]. There were six tasks yielding a maximum of 35 points, with higher scores implying better functioning. A description of the TICS supporting the definition of cognitive impairment can be found elsewhere [23-25]. In short, 8 points or less identifies the population with severe cognitive impairment. We explored the sensitivity of cut off points 7 and 9, which did not result in meaningful changes (results not shown). In about $10 \%$ of the sample in each wave, proxies represented respondents. Respondents whose memory and judgment were assessed as poor, were classified as cognitively impaired. We defined recovery as scoring 10 points or more, having been previously identified as cognitively impaired.

As behavioural risk factors, we studied self reported body mass index (BMI), smoking and levels of education. We categorized differences by ethnicity as non-Hispanic white (further called white), non-Hispanic black (further called black) and Hispanic. Self-reported weight and height at the first report defines BMI $(\mathrm{kg} / \mathrm{m} 2)$, classified as low normal weight (18.5-22.9), high normal weight (23-24.9), overweight (25-29.9), mildly obese (30-34.9) and severely obese $(35+)$. To avoid weight loss as a sign of impending disease, we excluded persons with a BMI less than 18.5. Smoking status is included as 'never smoked', 'stopped smoking' and 'currently smoking' based on the first reported information on smoking status. We distinguish three groups of educational attainment: Less than highschool or General Educational Development (GED), HighSchool graduate and some college, and College graduate and above. 
We used a multistate model for the analysis of transition rates to cognitive impairment, recovery and death (see Fig. 1). Lacking exact event dates of incidence and recovery, we assume these transitions to occur halfway between two biannual waves. An alternative assumption would be to assume a continuous-time Markov process, implying an exponential distribution of time of transition within the interval, but in intervals of 2 years, the difference between both assumptions is small [26]. Two separate statistical analyses were conducted: Cox proportional hazard analyses and multistate life table analyses. We estimated Cox proportional hazard models, including sampling weights to account for the oversampling of minority ethnic groups and used age as the time scale for the baseline hazard, consistent with the life table structure [27]. We used Schoenfeld residuals test to verify proportionality of the hazard model over age. Because the effect of the risk factors was not proportional for the transitions healthy to death or cognitive impairment, we ran the Cox analysis separately for ages 55-75 under and age 75+. These analyses result in proportional hazard ratios for each risk factor of interest with respect to a reference category. For the multistate life table analyses, we estimated baseline age-specific hazard rates of transitions to death and cognitive impairment for males, females and for each determinant separately. Rates estimated by years of age exhibit considerable random variation. The rates are therefore smoothed by fitting an exponential (Poisson) model through the age-specific rates. The exponential function fitted well. The multistate life table applies the estimated rates to a synthetic cohort and summarizes the age-specific transition rates into durations, here life expectancy at age 55 with or without cognitive impairment. Life expectancies by risk factor are only shown for the white population, as the sample of individuals of ethnic minorities by risk factor was too small. Confidence intervals for the multistate life table outcomes were calculated using bootstrapping with 250 replicates. The bootstrap procedure comprised both the estimation of the rates and the computation of the ensuing life expectancies.

\section{Results}

From the HRS sample $(N=30,207)$, we selected individuals who participated at least 2 waves $(N=24,586)$, reported information on BMI, smoking and education $(23,817)$, and had a BMI $>18.5(23,408)$ and were aged 55 or over, resulting in a sample of $22,388,9,834$ males and 12,554 females.

Table 1 shows the characteristics of the study population. 861 men and 1,332 women experienced the onset of cognitive impairment during observation, of which $39 \%$
Table 1 Distribution of sample characteristics at entry into the survey

\begin{tabular}{|c|c|c|c|c|}
\hline \multirow[b]{2}{*}{ Total number of individuals } & \multicolumn{2}{|l|}{ Males } & \multicolumn{2}{|l|}{ Females } \\
\hline & 9,834 & $100 \%$ & 12,554 & $100 \%$ \\
\hline Total personyears & 78,180 & & 98,674 & \\
\hline Incidence of cognitive impairment & 861 & & 1,332 & \\
\hline Mortality (all causes) & 2,446 & & 2,409 & \\
\hline \multicolumn{5}{|l|}{ Race/ethnicity } \\
\hline White & 7,763 & $79 \%$ & 9,579 & $76 \%$ \\
\hline Black & 1,309 & $13 \%$ & 1,985 & $16 \%$ \\
\hline Hispanic & 762 & $8 \%$ & 990 & $8 \%$ \\
\hline \multicolumn{5}{|l|}{ Education } \\
\hline Low education & 3,409 & $35 \%$ & 4,258 & $34 \%$ \\
\hline Medium education & 4,446 & $45 \%$ & 6,682 & $53 \%$ \\
\hline High education & 1,979 & $20 \%$ & 1,614 & $13 \%$ \\
\hline \multicolumn{5}{|l|}{ Smoking } \\
\hline Never smoked & 2,568 & $26 \%$ & 6,592 & $53 \%$ \\
\hline Stopped smoking & 5,036 & $51 \%$ & 3,731 & $30 \%$ \\
\hline Currently smoking & 2,230 & $23 \%$ & 2,231 & $18 \%$ \\
\hline \multicolumn{5}{|l|}{ BMI } \\
\hline BMI 18.5-23 & 1,287 & $13 \%$ & 3,075 & $24 \%$ \\
\hline BMI 23-25 & 1,800 & $18 \%$ & 2,189 & $17 \%$ \\
\hline BMI 25-30 & 4,804 & $49 \%$ & 4,357 & $35 \%$ \\
\hline BMI 30-35 & 1,516 & $15 \%$ & 1,975 & $16 \%$ \\
\hline BMI 35+ & 427 & $4 \%$ & 958 & $8 \%$ \\
\hline \multicolumn{5}{|l|}{ Age at entry } \\
\hline$[55,65]$ & 6,045 & $61 \%$ & 7,013 & $56 \%$ \\
\hline$[65,75]$ & 2,424 & $25 \%$ & 3,206 & $26 \%$ \\
\hline$[75,85]$ & 1,143 & $12 \%$ & 1,849 & $15 \%$ \\
\hline$[85,95]$ & 216 & $2 \%$ & 459 & $4 \%$ \\
\hline$[95,105]$ & 6 & $0 \%$ & 27 & $0 \%$ \\
\hline
\end{tabular}

and $35 \%$ reported by proxy. A total of 557 individuals experienced recovery, $28 \%$ and $23 \%$ for males and females respectively. We ignored recovery in multiple relapse/ recovery/relapse episodes as these were rare $(N=26)$.

Table 2 shows the proportional hazard ratios for transitions to death and to cognitive impairment by race, BMI, smoking status and levels of education. The effects of risk factors on recovery from cognitive impairment to healthy were small (results not shown). The risk to cognitive impairment is about twice as high for blacks and Hispanics as for whites (not significant for Hispanics aged 75+). BMI has little effect on the incidence of cognitive impairment or on the survival of the impaired. BMI over 35 increases the risk of cognitive impairment for males. This effect disappears when we control for interaction effects with race, smoking and education. The evidence of an effect of smoking is mixed and disappears after including interaction effects (results not shown). Higher education delays incidence of cognitive decline in both males and females. 
Table 2 Cox proportional hazard ratios by risk factor status (95\% confidence intervals), adjusted for each other

\begin{tabular}{|c|c|c|c|c|}
\hline & \multicolumn{2}{|l|}{ Males } & \multicolumn{2}{|l|}{ Females } \\
\hline & $55-74$ & $75+$ & $55-74$ & $75+$ \\
\hline \multicolumn{5}{|l|}{ Healthy to death } \\
\hline White & 1.00 & 1.00 & 1.00 & 1.00 \\
\hline Black & $1.30(1.09 ; 1.55)$ & $1.16(0.93 ; 1.44)$ & $1.35(1.10 ; 1.65)$ & $1.23(1.00 ; 1.52)$ \\
\hline Hispanic & $0.77(0.53 ; 1.11)$ & $0.90(0.59 ; 1.38)$ & $0.99(0.66 ; 1.48)$ & $1.03(0.63 ; 1.66)$ \\
\hline BMI 18.5-22.9 & 1.39 (1.16; 1.67) & $1.27(1.07 ; 1.51)$ & $0.83(0.67 ; 1.02)$ & $1.04(0.88 ; 1.22)$ \\
\hline BMI 23-24.9 & 1.00 & 1.00 & 1.00 & 1.00 \\
\hline BMI 25-29.9 & $0.89(0.76 ; 1.03)$ & $0.87(0.75 ; 1.01)$ & $0.90(0.75 ; 1.09)$ & $0.91(0.77 ; 1.07)$ \\
\hline BMI 30-34.9 & $1.14(0.95 ; 1.37)$ & $1.01(0.81 ; 1.25)$ & $0.98(0.77 ; 1.23)$ & $0.93(0.76 ; 1.15)$ \\
\hline BMI 35+ & $1.45(1.09 ; 1.91)$ & $1.23(0.83 ; 1.83)$ & $1.55(1.21 ; 1.99)$ & $1.22(0.89 ; 1.66)$ \\
\hline Never smoked & 1.00 & 1.00 & 1.00 & 1.00 \\
\hline Stopped smoking & $1.42(1.21 ; 1.66)$ & $1.25(1.08 ; 1.44)$ & $1.37(1.17 ; 1.60)$ & $1.21(1.06 ; 1.37)$ \\
\hline Currently smoking & $2.67(2.25 ; 3.17)$ & $1.67(1.37 ; 2.03)$ & $2.57(2.18 ; 3.03)$ & $1.85(1.52 ; 2.24)$ \\
\hline Low education & 1.00 & 1.00 & 1.00 & 1.00 \\
\hline Medium education & $0.93(0.82 ; 1.05)$ & $0.91(0.80 ; 1.03)$ & $0.70(0.61 ; 0.81)$ & $0.83(0.74 ; 0.94)$ \\
\hline High education & $0.62(0.52 ; 0.73)$ & $0.65(0.54 ; 0.78)$ & $0.47(0.36 ; 0.61)$ & $0.85(0.69 ; 1.04)$ \\
\hline \multicolumn{5}{|l|}{ Healthy to CI } \\
\hline White & 1.00 & 1.00 & 1.00 & 1.00 \\
\hline Black & $2.96(2.27 ; 3.86)$ & $1.83(1.23 ; 2.71)$ & $2.92(2.22 ; 3.84)$ & $2.06(1.55 ; 2.72)$ \\
\hline Hispanic & 1.85 (1.09; 3.12) & $1.76(0.88 ; 3.50)$ & $2.11(1.32 ; 3.36)$ & $1.50(0.75 ; 2.99)$ \\
\hline BMI 18.5-22.9 & $1.63(1.07 ; 2.51)$ & $1.00(0.70 ; 1.44)$ & $1.13(0.72 ; 1.77)$ & $1.11(0.84 ; 1.47)$ \\
\hline BMI 23-24.9 & 1.00 & 1.00 & 1.00 & 1.00 \\
\hline BMI 25-29.9 & $1.10(0.77 ; 1.58)$ & $0.70(0.51 ; 0.95)$ & $1.34(0.90 ; 1.99)$ & $0.93(0.71 ; 1.23)$ \\
\hline BMI 30-34.9 & $1.27(0.84 ; 1.92)$ & $0.65(0.40 ; 1.07)$ & $1.36(0.88 ; 2.10)$ & $0.89(0.63 ; 1.26)$ \\
\hline BMI 35+ & $1.71(1.00 ; 2.92)$ & $0.66(0.24 ; 1.82)$ & $1.41(0.87 ; 2.28)$ & $0.89(0.50 ; 1.58)$ \\
\hline Never smoked & 1.00 & 1.00 & 1.00 & 1.00 \\
\hline Stopped smoking & $0.69(0.52 ; 0.92)$ & $1.02(0.76 ; 1.37)$ & $1.45(1.10 ; 1.90)$ & $0.86(0.69 ; 1.07)$ \\
\hline Currently smoking & $0.93(0.69 ; 1.26)$ & $0.88(0.55 ; 1.39)$ & $1.26(0.93 ; 1.70)$ & $0.63(0.39 ; 1.01)$ \\
\hline Low education & 1.00 & 1.00 & 1.00 & 1.00 \\
\hline Medium education & $0.50(0.39 ; 0.64)$ & $0.53(0.40 ; 0.70)$ & $0.36(0.28 ; 0.46)$ & $0.65(0.53 ; 0.79)$ \\
\hline High education & $0.14(0.09 ; 0.24)$ & $0.42(0.28 ; 0.63)$ & $0.25(0.15 ; 0.40)$ & $0.49(0.33 ; 0.73)$ \\
\hline \multicolumn{5}{|l|}{ CI to death } \\
\hline White & 1.00 & 1.00 & 1.00 & 1.00 \\
\hline Black & $0.72(0.37 ; 1.40)$ & $0.74(0.49 ; 1.12)$ & $1.37(0.73 ; 2.57)$ & $0.80(0.55 ; 1.15)$ \\
\hline Hispanic & $0.90(0.32 ; 2.56)$ & $0.42(0.16 ; 1.14)$ & $0.91(0.22 ; 3.72)$ & $0.76(0.35 ; 1.65)$ \\
\hline BMI 18.5-22.9 & $1.56(0.66 ; 3.67)$ & $0.99(0.65 ; 1.52)$ & $0.97(0.42 ; 2.21)$ & $1.25(0.86 ; 1.81)$ \\
\hline BMI 23-24.9 & 1.00 & 1.00 & 1.00 & 1.00 \\
\hline BMI 25-29.9 & $0.82(0.36 ; 1.83)$ & $0.79(0.53 ; 1.17)$ & $0.62(0.27 ; 1.41)$ & $0.95(0.63 ; 1.43)$ \\
\hline BMI 30-34.9 & $1.89(0.71 ; 5.01)$ & $0.94(0.50 ; 1.79)$ & $0.60(0.24 ; 1.51)$ & $1.24(0.76 ; 2.01)$ \\
\hline BMI 35+ & 3.33 (1.22; 9.07) & $1.26(0.50 ; 3.17)$ & $0.48(0.14 ; 1.60)$ & $1.60(0.73 ; 3.51)$ \\
\hline Never smoked & 1.00 & 1.00 & 1.00 & 1.00 \\
\hline Stopped smoking & $1.23(0.60 ; 2.53)$ & $1.06(0.74 ; 1.53)$ & $1.14(0.59 ; 2.19)$ & $1.16(0.84 ; 1.60)$ \\
\hline Currently smoking & $2.04(1.01 ; 4.10)$ & $1.28(0.76 ; 2.17)$ & $1.10(0.55 ; 2.18)$ & $1.38(0.76 ; 2.51)$ \\
\hline Low education & 1.00 & 1.00 & 1.00 & 1.00 \\
\hline Medium education & $1.17(0.62 ; 2.21)$ & $1.22(0.85 ; 1.75)$ & $3.36(1.83 ; 6.17)$ & $1.44(1.08 ; 1.92)$ \\
\hline High education & $2.08(0.84 ; 5.17)$ & $1.29(0.68 ; 2.43)$ & $1.33(0.40 ; 4.45)$ & $1.40(0.77 ; 2.56)$ \\
\hline
\end{tabular}

Significant ratios are printed in bold 
Table 3 Life years to live at age 55 with or without cognitive impairment $(\mathrm{CI})$ by ethnic group

limits

\begin{tabular}{llll}
\hline & Life expectancy & Life years with CI & Life years without CI \\
\hline Men & & & \\
All & $25.13(24.74 ; 25.52)$ & $2.53(2.32 ; 2.74)$ & $22.60(22.24 ; 22.96)$ \\
White & $25.44(25.04 ; 25.84)$ & $1.69(1.50 ; 1.89)$ & $23.75(23.34 ; 24.15)$ \\
Black & $22.86(21.86 ; 23.87)$ & $5.42(4.54 ; 6.30)$ & $17.44(16.55 ; 18.34)$ \\
Hispanic & $27.04(25.37 ; 28.70)$ & $4.94(3.61 ; 6.27)$ & $22.10(20.69 ; 23.51)$ \\
Women & & & \\
All & $29.43(29.06 ; 29.80)$ & $3.77(3.52 ; 4.02)$ & $25.66(25.31 ; 26.01)$ \\
White & $30.01(29.59 ; 30.44)$ & $2.66(2.43 ; 2.89)$ & $27.35(26.96 ; 27.75)$ \\
Black & $26.54(25.64 ; 27.44)$ & $6.38(5.65 ; 7.12)$ & $20.16(19.37 ; 20.94)$ \\
Hispanic & $31.94(30.16 ; 33.72)$ & $8.50(6.88 ; 10.1)$ & $23.44(22.08 ; 24.80)$ \\
\hline
\end{tabular}

The mortality risks of higher educated individuals, once cognitively impaired, tend to be higher compared to the lower educated, although this is only significant for medium educated females. Higher education protects more against cognitive impairment between age 55 and 75 than at 75 and over, the effect of education is stronger for blacks than for whites: hazard ratios to cognitive impairment for black men and women are 0.09 [95\% confidence intervals $0.01 ; 1.48]$ and $0.10[0.01 ; 0.73]$ compared to 0.28 [0.16; $0.48]$ and $0.25[0.11 ; 0.58]$ for whites.

Translating age, sex and risk factor-specific transition rates into life expectancies at age 55 defines the stratified life expectancy with and without cognitive impairment for each risk group. The life expectancy of our study population was respectively 25.4 and 30.0 years for whites, 22.9 and 26.5 for blacks and 27.0 and 31.9 for Hispanics (see Table 3). Average lifespan with cognitive impairment differs remarkably between the ethnic groups. In the white population, 55 year old males and females spent respectively $1.7[1.5 ; 1.9]$ years and $2.7[2.4 ; 2.9]$ years with cognitive impairment. Although their life expectancy was shorter, male and female blacks lived on average 3.7 [2.9; 4.5] years longer with cognitive impairment than whites (see Table 2 and Fig. 2). Blacks lived more than 5 year shorter free of cognitive impairment. Hispanic men and women lived $3.2[1.9 ; 4.6]$ and $5.8[4.2 ; 7.5]$ years more with cognitive impairment compared to white men and women. Hispanics lived longer than whites, but shorter free of cognitive impairment.

The number of years lived with cognitive impairment varied among risk factors (see Table 4 and Fig. 2). Data are only shown for the white population in Table 4. BMI does not change duration of life with cognitive impairment. Smoking shortens the duration of cognitive impairment. Ever and current smokers live significantly shorter than never smokers with cognitive impairment: -0.7 years $[-1.2 ;-0.3]$ for men and $-0.9[-1.3 ;-0.5]$ for women. The high mortality of smoking compresses both morbidity and a healthy life [28-30]. A high education effectively

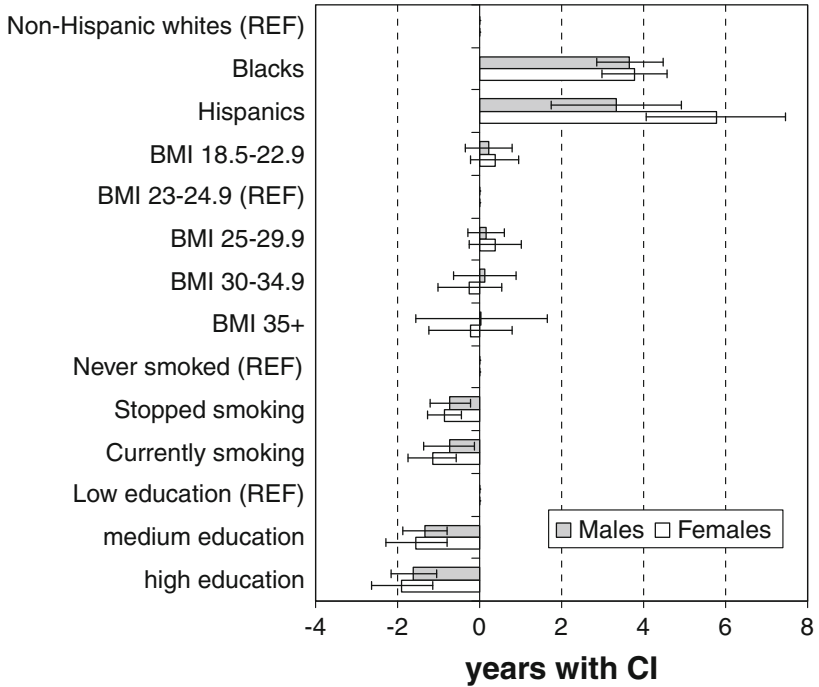

Fig. 2 Gains and losses in years lived with cognitive impairment (CI) by characteristics. $B M I$, smoking and education show the effects for non-Hispanic whites. Errors bars are 95\% confidence intervals

and seriously compresses cognitive impairment. Highly educated men live 1.1 years $[0.7 ; 1.4]$ with cognitive impairment, which is 1.6 years $[1.1 ; 2.2]$ shorter than lowly educated men. Highly educated women, live 1.9 years [1.4; 2.4] with cognitive impairment, which is 1.9 years [1.6; 2.6] shorter than lowly educated women. For black men and women, low education doubles the number of years with cognitive impairment from $3.2[2.1 ; 4.4]$ and $4.0[3.0$; 5.1] for medium or high education to $6.8[5.6 ; 7.9]$ and 8.2 [7.1; 9.3] for lowly educated.

Factors that extend lifespan to older ages, like being a woman or a non-smoker, increase the duration of cognitive impairment. Only higher levels of education increase life in good cognitive health while decreasing the duration of cognitive impairment. Ironically, lifespan with cognitive impairment is most compressed in the highly educated smokers. Figure 2 shows the gains and losses in years lived with cognitive impairment first by ethnic belonging and 
Table 4 Life years to live at age 55 with or without cognitive impairment (CI) by risk factor status

\begin{tabular}{|c|c|c|c|}
\hline & Life expectancy & Life years with CI & Life years without CI \\
\hline \multicolumn{4}{|l|}{ White men } \\
\hline BMI 18.5-22.9 & $22.95(21.82 ; 24.07)$ & $1.82(1.35 ; 2.28)$ & $21.13(20.10 ; 22.16)$ \\
\hline BMI 23-24.9 & $25.55(24.68 ; 26.43)$ & $1.59(1.25 ; 1.92)$ & 23.97 (23.07; 24.87) \\
\hline BMI 25-29.9 & $26.54(25.90 ; 27.18)$ & $1.73(1.43 ; 2.03)$ & $24.81(24.19 ; 25.42)$ \\
\hline BMI 30-34.9 & $24.93(23.84 ; 26.03)$ & $1.71(1.01 ; 2.41)$ & $23.22(22.18 ; 24.27)$ \\
\hline BMI 35+ & $23.02(20.75 ; 25.29)$ & $1.63(0.06 ; 3.20)$ & $21.39(19.58 ; 23.21)$ \\
\hline Never smoked & $29.12(28.23 ; 30.02)$ & $2.25(1.81 ; 2.70)$ & $26.87(26.02 ; 27.72)$ \\
\hline Stopped smoking & $26.16(25.62 ; 26.70)$ & $1.54(1.35 ; 1.73)$ & $24.62(24.09 ; 25.16)$ \\
\hline Currently smoking & $20.34(19.48 ; 21.21)$ & $1.52(1.09 ; 1.95)$ & $18.82(18.04 ; 19.60)$ \\
\hline Low education & $23.60(22.84 ; 24.36)$ & $2.65(2.20 ; 3.11)$ & $20.95(20.22 ; 21.67)$ \\
\hline Medium education & $25.16(24.58 ; 25.75)$ & $1.32(1.05 ; 1.59)$ & $23.84(23.27 ; 24.42)$ \\
\hline High education & $28.85(27.90 ; 29.81)$ & $1.05(0.73 ; 1.36)$ & $27.80(26.88 ; 28.72)$ \\
\hline \multicolumn{4}{|l|}{ White women } \\
\hline BMI 18.5-22.9 & $30.22(29.49 ; 30.95)$ & $2.82(2.13 ; 3.51)$ & $27.40(26.71 ; 28.09)$ \\
\hline BMI 23-24.9 & $30.12(29.32 ; 30.92)$ & $2.45(2.01 ; 2.88)$ & $27.68(26.86 ; 28.49)$ \\
\hline BMI 25-29.9 & $30.70(29.93 ; 31.47)$ & $2.82(2.36 ; 3.28)$ & $27.88(27.20 ; 28.56)$ \\
\hline BMI 30-34.9 & $29.87(28.80 ; 30.94)$ & $2.58(1.93 ; 3.23)$ & $27.29(26.25 ; 28.33)$ \\
\hline BMI 35+ & $25.90(24.12 ; 27.69)$ & $2.21(1.30 ; 3.13)$ & $23.69(22.08 ; 25.30)$ \\
\hline Never smoked & $31.89(31.37 ; 32.42)$ & $3.00(2.72 ; 3.29)$ & $28.89(28.43 ; 29.34)$ \\
\hline Stopped smoking & $29.61(28.96 ; 30.26)$ & $2.16(1.85 ; 2.46)$ & $27.46(26.77 ; 28.14)$ \\
\hline Currently smoking & $24.90(23.96 ; 25.84)$ & $1.85(1.34 ; 2.35)$ & $23.06(22.13 ; 23.98)$ \\
\hline Low education & $28.01(27.19 ; 28.83)$ & 3.78 (3.28; 4.29) & $24.23(23.48 ; 24.97)$ \\
\hline Medium education & $30.60(30.06 ; 31.15)$ & $2.24(2.00 ; 2.48)$ & $28.36(27.87 ; 28.86)$ \\
\hline High education & $31.88(30.88 ; 32.89)$ & $1.89(1.36 ; 2.42)$ & $29.99(29.09 ; 30.90)$ \\
\hline
\end{tabular}

In brackets $95 \%$ confidence limits

then by risk factors for whites, illustrating the distinctive effect of smoking and education. Smoking shortens life with and without cognitive impairment. Higher education extends life in cognitive health and compresses cognitive impairment to a shorter period at the end of life.

A measure of interest both for individuals and public health, is the cumulative probability of ever developing cognitive impairment before death.

A high probability is often the prize paid for a long life. In the HRS life table cohort of white individuals at age 55, more than one in three women $(36 \%[0.34 \%, 0.38 \%])$ will experience cognitive impairment compared to close to one in four men $(23 \%[0.21 \%, 0.24 \%])$ (See Table 5).

Blacks face a higher cumulative probability of cognitive impairment: respectively $44 \%$ [39\%; 48\%] and 53\% [49\%; 57\%] for men and women. So do Hispanics: 46\% [39\%; $54 \%$ ] and $61 \%$ [54\%; 68\%] for men and women. BMI has little effect on the cumulative probability of cognitive impairment, but smoking considerably lowers the life time risk of cognitive impairment at age 55: from $28 \%$ [23\%; $0.32 \%$ ] and $41 \%$ [38\%; $43 \%$ ] for never smoking white men and women to $18 \%[15 \% ; 22 \%]$ and $22 \%[17 \% ; 27 \%]$ smokers. Smoking kills many before the onset of cognitive
Table 5 Cumulative probability of cognitive impairment (95\% confidence bands) at age 55

\begin{tabular}{lll}
\hline & Males & Females \\
\hline White & $22.6 \%(20.7 \% ; 24.5 \%)$ & $35.9 \%(33.7 \% ; 38.1 \%)$ \\
Black & $43.9 \%(38.9 \% ; 47.7 \%)$ & $53.3 \%(49.0 \% ; 56.5 \%)$ \\
Hispanic & $46.2 \%(39.1 \% ; 54.3 \%)$ & $60.8 \%(53.6 \% ; 68.1 \%)$ \\
BMI 18.5-22.9 & $24.2 \%(20.0 \% ; 28.5 \%)$ & $37.8 \%(34.3 \% ; 41.4 \%)$ \\
BMI 23-24.9 & $22.5 \%(18.8 \% ; 26.3 \%)$ & $36.2 \%(31.7 \% ; 40.6 \%)$ \\
BMI 25-29.9 & $22.2 \%(19.4 \% ; 25.0 \%)$ & $35.6 \%(32.4 \% ; 38.8 \%)$ \\
BMI 30-34.9 & $20.6 \%(14.9 \% ; 26.3 \%)$ & $34.7 \%(28.4 \% ; 41.1 \%)$ \\
BMI 35+ & $21.6 \%(10.3 \% ; 33.0 \%)$ & $28.4 \%(20.4 \% ; 36.5 \%)$ \\
Never smoked & $28.0 \%(23.4 \% ; 32.5 \%)$ & $40.7 \%(38.0 \% ; 43.3 \%)$ \\
Stopped smoking & $22.0 \%(19.9 \% ; 24.1 \%)$ & $31.8 \%(28.2 \% ; 35.4 \%)$ \\
Currently smoking & $18.4 \%(15.0 \% ; 21.8 \%)$ & $22.0 \%(17.4 \% ; 26.6 \%)$ \\
Low education & $29.6 \%(26.2 \% ; 33.1 \%)$ & $39.2 \%(35.7 \% ; 42.6 \%)$ \\
Medium education & $17.6 \%(14.6 \% ; 20.7 \%)$ & $35.7 \%(32.7 \% ; 38.8 \%)$ \\
High education & $19.8 \%(12.9 \% ; 26.7 \%)$ & $29.3 \%(22.4 \% ; 36.2 \%)$ \\
\hline
\end{tabular}

$B M I$, smoking and education refer to white non-Hispanics only

impairment. High education increases life expectancy while decreasing the probability of cognitive impairment. The lifetime probability of cognitive impairment is lower 
in the higher educated men and women, for whites respectively $20 \%[13 \% ; 27 \%]$ and $29 \%[22 \% ; 36 \%])$ compared to the lower educated people, respectively $30 \%$ [26\%; 33\%] among men and 39\% [36\%; 43\%] among women.

When expressed as relative percentages of total life expectancy, a long life goes with a relatively long duration of cognitive impairment. White men live on average $6.7 \%$ [5.9\%; 7.4\%] of their life after age 55 with cognitive impairment; white women $8.9 \%$ [8.2\%; 9.6\%]. Black men and women can expect to live $23.7 \%$ [20.3\%; $27.1 \%]$ and $24.1 \%[21.6 \% ; 26.5 \%]$ of their life over age 55 with cognitive impairment, Hispanics $18.3 \%$ [13.9\%; 22.6\%] and $26.6 \%[22.4 \% ; 30.8 \%]$. Again education has a significant impact on this share, ranging from $11.2 \%$ [9.4\%; $13.1 \%]$ and $3.6 \%[2.6 \% ; 4.7 \%]$ for respectively lowly and highly educated white men and from $13.5 \%$ [11.8\%; $15.2 \%]$ to $5.9 \%[4.2 \% ; 7.7 \%]$ for lowly and highly educated white women.

\section{Discussion}

The life table cohort of Americans aged 55 and over from the HRS study shows that white race, male sex, smoking and higher education shorten duration of life with cognitive impairment. Blacks have both shorter life expectancies, and they live more life years with cognitive impairment. Hispanics live longer with cognitive impairment. Women and never smokers live more years with cognitive impairment than men and smokers, simply because they live longer. Self reported BMI had no notable effect on the duration of cognitive impairment. High education increased total lifespan, but increased lifespan free of cognitive decline even more, shortening life with cognitive impairment.

The life expectancy of the US population in 2003 was 24.6 and 28.1 for white men and women and 21.2 and 25.9 for black men and women at age 55 [31]. Calculated life expectancies of the HRS were slightly higher, indicating that the studied population was slightly healthier than the source population. We excluded small numbers of people with BMI under 18.5 and the HRS sample includes only non-institutionalized persons at baseline, but follows these into long term care institutions. Estimates of life years with cognitive impairment will therefore be slightly underestimated.

Our findings of a short duration of cognitive impairment in highly educated men and women agree with the cognitive reserve hypothesis [32-34]. The cognitive reserve hypothesis posits that a higher cognitive reserve leads to a more plastic and adaptable brain, supporting more neuropathological and vascular damage before expressing clinical dementia. Recently, the Epidemiological Clinicopathological Studies in Europe (ELIPSE); confirmed that longer years in education were associated with a decreased dementia risk but had no relationship to neurodegenerative or vascular pathologies [35]. The 'dose' of education reduced dementia risk independently of severity of pathology. More education did not protect individuals from developing neurodegenerative and vascular neuropathology by the time they died but it mitigated the impact of pathology on the clinical expression of dementia before death [35]. The protective effect of high education among African Americans was even stronger.

Our findings of increased incidence and duration of cognitive impairment in ethnic minority groups, in the US black and Hispanics, are consistent with other epidemiologic studies describing higher prevalence and incidence of dementia in racial and ethnic minorities [15-17, 36]. We identified many reasons why the observed occurrence of dementia may appear higher in ethnic minorities than in whites in the HRS. Culturally and educationally sensitive diagnostic ascertainment methods lead to very different estimates of dementia prevalence in low and middle income countries [37]. In less educated minority populations, the MMSE (source of the TICS used in the HRS) is less specific and yields lower scores [18, 38]. Differences in learned test-taking strategies, comfort with testing staff, and cultural relevance of test items to the interviewees explain some of the race differences [39].Differences in levels of education between majority and minority groups may not fully be accounted for by actual stratification in three classes [36, 38]. Finally, prevalence of uncontrolled hypertension and diabetes, known causes of dementia, is higher in African Americans $[17,40-42]$. A literature survey offered no information on European ethnic minorities from Northern Africa or Turkey.

We found little effect of BMI on the incidence of cognitive impairment. However, weight loss induced by imminent disease masks effects of increased BMI over the life span, a suspected cause of dementia [2, 43, 44]. The disentanglement of the relationships of BMI, weigh loss and cognitive impairment is beyond the scope of this study. Consistent with a long history of debate of smoking as a risk factor for dementia [14], we found mixed results for the relation between smoking and cognitive impairment. However, any increased risk of cognitive impairment is largely offset by the high mortality of smokers, shortening life expectancy with cognitive impairment.

The HRS is a comprehensive social study, not a specific epidemiological study that provides a clinical diagnosis of dementia. The definition of cognitive impairment has to be understood as a score of a screening tool, comparable to the MMSE, which is correlated to, but not identical with clinical diagnoses of cognitive impairment or dementia [45]. A certain weakness of this study is the lack of detail 
of other known causal factors related to the onset of dementia, high blood pressure, moderate and high alcohol consumption, a history of diabetes and the presence of the apolipoprotein E $\varepsilon 4$ allele. The main reason was to keep the MSLT analysis parsimonious, focusing on few determinants. We excluded alcohol use, as drinking 6 glasses or more per day showed a protective effect on incidence of cognitive impairment (results not shown). While moderate alcohol use may protect against dementia, it is not plausible that this holds for high levels of use [46, 47].

The results are to be interpreted as a period life table, a demographic tool that reduces very many age specific event rates to few and intuitively transparent durations. Event rates over a period of 12 year of follow up in a population are applied to a synthetic cohort from age 55 till extinction. This illustrates a general problem of life courses: true cohorts running till old age yield information from long ago, period data are recent but collapse many cohorts of a population into one single imaginary cohort.

Cognitive impairment is a major cause of disability and care dependence. All people fear loss of cognition and the ability for self care nearly as much as death itself. Maybe the most distressing results were the very large differences for the ethnic minority populations of the USA. We found no information on incidence and prevalence of dementia in European migrant populations, maybe because old age is still relatively infrequent. However, our migrant populations share many characteristics of the US black population: a lower education, higher risks of social deprivation and a high prevalence of diabetes [48]. Lacking any information on the mental health of migrant elderly in Europe, it seems safer to expect similar consequences from similar characteristics. As our migrant populations start aging, this is a most important research agenda. Obesity showed little effects, smoking reduced morbidity, but by killing early. The most promising result of this study was that high levels of education not only extend life and extend life in good health, but truly compress cognitive impairment by postponing incidence of dementia more than death. Raising education to the highest level attainable in a society is therefore not only a millennium goal for developing countries. The best investments in future health remain investments in the best education today. If we may extrapolate from the ethnic minorities of the USA, this will hold particularly for our younger migrant populations.

Acknowledgments This research was funded by the Royal Netherlands Academy of Science funding for research on Demography, Epidemiology and Actuarial science. The Health and Retirement Study is a public use dataset, produced and distributed by the University of Michigan with funding from the National Institute on Aging (grant number NIA U01AG009740). Ann Arbor, MI, (2006). This study makes use of the RAND HRS version F.
Open Access This article is distributed under the terms of the Creative Commons Attribution Noncommercial License which permits any noncommercial use, distribution, and reproduction in any medium, provided the original author(s) and source are credited.

\section{References}

1. Jorm AF, Korten AE, Henderson AS. The prevalence of dementia: a quantitative integration of the literature. Acta Psychiatr Scand. 1987;76(5):465-79.

2. Qiu C, De Ronchi D, Fratiglioni L. The epidemiology of the dementias: an update. Curr Opin Psychiatry. 2007;20(4):380-5.

3. Ferri CP, Prince M, Brayne C, Brodaty H, Fratiglioni L, Ganguli $\mathrm{M}$, et al. Global prevalence of dementia: a Delphi consensus study. Lancet. 2005;366(9503):2112-7.

4. Rogers RG, Rogers A, Belanger A. Active life among the elderly in the United States: multistate life-table estimates and population projections. Milbank Q. 1989;67(3):370-411.

5. Sullivan DF. A single index of mortality and morbidity. HSMHA Health Rep. 1971;86(4):347.

6. Barendregt J, Bonneux L, Van der Maas P. Health expectancy: an indicator for change? Technology Assessment Methods Project Team. J Epidemiol Community Health. 1994;48(5):482-7.

7. Zhu L, Fratiglioni L, Guo Z, Basun H, Corder EH, Winblad B, et al. Incidence of dementia in relation to stroke and the apolipoprotein E epsilon4 allele in the very old. Findings from a population-based longitudinal study. Stroke. 2000;31(1):53-60.

8. van Oijen M, de Jong FJ, Witteman JC, Hofman A, Koudstaal PJ, Breteler MM. Atherosclerosis and risk for dementia. Ann Neurol. 2007;61(5):403-10.

9. Biessels GJ, Staekenborg S, Brunner E, Brayne C, Scheltens P. Risk of dementia in diabetes mellitus: a systematic review. Lancet Neurol. 2006;5(1):64-74.

10. Hofman A, de Jong PT, van Duijn CM, Breteler MM. Epidemiology of neurological diseases in elderly people: what did we learn from the Rotterdam Study? Lancet Neurol. 2006;5(6): 545-50.

11. Qiu C, Winblad B, Fratiglioni L. The age-dependent relation of blood pressure to cognitive function and dementia. Lancet Neurol. 2005;4(8):487-99.

12. Ruitenberg A, den Heijer T, Bakker SL, van Swieten JC, Koudstaal PJ, Hofman A, et al. Cerebral hypoperfusion and clinical onset of dementia: the Rotterdam Study. Ann Neurol. 2005;57(6):789-94.

13. Gustafson D. Adiposity indices and dementia. Lancet Neurol. 2006;5(8):713-20.

14. Anstey KJ, Sanden VC, Salim A, O'Kearney R. Smoking as a risk factor for dementia and cognitive decline: a meta-analysis of prospective studies. Am J Epidemiol. 2007;166(4):367-78.

15. Cooper C, Tandy AR, Balamurali TB, Livingston G. A systematic review and meta-analysis of ethnic differences in use of dementia treatment, care, and research. Am J Geriatr Psychiatry. 18(3):193-203.

16. Bachman DL, Green RC, Benke KS, Cupples LA, Farrer LA. Comparison of Alzheimer's disease risk factors in white and African American families. Neurology. 2003;60(8):1372-4.

17. Tang MX, Cross P, Andrews H, Jacobs DM, Small S, Bell K, et al. Incidence of $\mathrm{AD}$ in African-Americans, Caribbean Hispanics, and Caucasians in northern Manhattan. Neurology. 2001;56(1):49-56.

18. Ng TP, Niti M, Chiam PC, Kua EH. Ethnic and educational differences in cognitive test performance on mini-mental state examination in Asians. Am J Geriatr Psychiatry. 2007;15(2): $130-9$. 
19. Mehta KM, Yaffe K, Perez-Stable EJ, Stewart A, Barnes D, Kurland BF, et al. Race/ethnic differences in AD survival in US Alzheimer's Disease Centers. Neurology. 2008;70(14):1163-70.

20. Glymour MM, Manly JJ. Lifecourse social conditions and racial and ethnic patterns of cognitive aging. Neuropsychol Rev. 2008;18(3):223-54.

21. Brandt J, Spencer M, Folstein M. The telephone interview for cognitive status. Neuropsychiatry Neuropsychol Behav Neurol. 1988;1(2):111-7.

22. Folstein MF, Robins LN, Helzer JE. The mini-mental state examination. Arch Gen Psychiatry. 1983;40(7):812.

23. Herzog AR, Wallace RB. Measures of cognitive functioning in the AHEAD Study. J Gerontol B Soc Sci. 1997;52(Spec No): 37-48.

24. Langa KM, Larson EB, Karlawish JH, Cutler DM, Kabeto MU, Kim SY, et al. Trends in the prevalence and mortality of cognitive impairment in the United States: Is there evidence of a compression of cognitive morbidity? Alzheimer's Dementia. 2008;4(2):134-44.

25. Suthers K, Kim JK, Crimmins E. Life expectancy with cognitive impairment in the older population of the United States. J Gerontol B Psychol Sci Soc Sci. 2003;58B(3):S179-86.

26. Izmirlian G, Brock D, Ferrucci L, Phillips C. Active life expectancy from annual follow-up data with missing responses. Biometrics. 2000;56(1):244-8.

27. Thiebaut AC, Benichou J. Choice of time-scale in Cox's model analysis of epidemiologic cohort data: a simulation study. Stat Med. 2004;23(24):3803-20.

28. Barendregt JJ, Bonneux L, van der Maas PJ. The health care costs of smoking. N Engl J Med. 1997;337(15):1052-7.

29. Mamun AA, Peeters A, Barendregt J, Willekens F, Nusselder W, Bonneux L. Smoking decreases the duration of life lived with and without cardiovascular disease: a life course analysis of the Framingham Heart Study. Eur Heart J. 2004;25(5):409-15.

30. Reuser M, Bonneux LG, Willekens FJ. Smoking kills, obesity disables: a multistate approach of the US health and retirement survey. Obesity. 2009;17(4):783-9.

31. Centers for Disease Control and Prevention, National Center for Health Statistics. National Vital Statistics Reports. 2006;54(14).

32. Fratiglioni L, Wang HX. Brain reserve hypothesis in dementia. J Alzheimers Dis. 2007;12(1):11-22.

33. Stern Y. Cognitive reserve and Alzheimer disease. Alzheimer Dis Assoc Disord. 2006;20(2):112-7.

34. Stern Y, Tang MX, Denaro J, Mayeux R. Increased risk of mortality in Alzheimer's disease patients with more advanced educational and occupational attainment. Ann Neurol. 1995;37:590-5.

35. Brayne C, Ince PG, Keage HAD, McKeith IG, Matthews FE, Polvikoski T, et al. Education, the brain and dementia: neuroprotection or compensation?: EClipSE Collaborative Members. Brain. 2010;133(8):2210-6.
36. Gurland BJ, Wilder DE, Lantigua R, Stern Y, Chen J, Killeffer $\mathrm{EH}$, et al. Rates of dementia in three ethnoracial groups. Int $\mathrm{J}$ Geriatr Psychiatry. 1999;14(6):481-93.

37. Llibre Rodriguez JJ, Ferri CP, Acosta D, Guerra M, Huang Y, Jacob KS, et al. Prevalence of dementia in Latin America, India, and China: a population-based cross-sectional survey. Lancet. 2008;372(9637):464-74.

38. Fitzpatrick AL, Kuller LH, Ives DG, Lopez OL, Jagust W, Breitner JC, et al. Incidence and prevalence of dementia in the Cardiovascular Health Study. J Am Geriatr Soc. 2004;52(2): 195-204.

39. Karlamangla AS, Miller-Martinez D, Aneshensel CS, Seeman TE, Wight RG, Chodosh J. Trajectories of cognitive function in late life in the United States: Demographic and socioeconomic predictors. Am J Epidemiol. 2009;170(3):334-42.

40. Natarajan S, Santa Ana EJ, Liao Y, Lipsitz SR, McGee DL. Effect of treatment and adherence on ethnic differences in blood pressure control among adults with hypertension. Ann Epidemiol. 2009;19(3):172-9.

41. Kramer H, Han C, Post W, Goff D, Diez-Roux A, Cooper R, et al. Racial/ethnic differences in hypertension and hypertension treatment and control in the multi-ethnic study of atherosclerosis (MESA). Am J Hypertens. 2004;17(10):963-70.

42. Maskarinec G, Grandinetti A, Matsuura G, Sharma S, Mau M, Henderson BE, et al. Diabetes prevalence and body mass index differ by ethnicity: the Multiethnic Cohort. Ethn Dis. 2009;19(1): $49-55$.

43. Gorospe EC, Dave JK. The risk of dementia with increased body mass index. Age Ageing. 2007;36(1):23-9.

44. Kivipelto M, Ngandu T, Fratiglioni L, Viitanen M, Kareholt I, Winblad B. Obesity and vascular risk factors at midlife and the risk of dementia and Alzheimer disease. Arch Neurol. 2005; 62(10): $1556-60$.

45. Langa K, Chernew M, Kabeto M, Herzog A, Ofstedal MB, Willis R. National estimates of the quantity and cost of informal caregiving for the elderly with dementia. J Gen Intern Med. $2001 ; 16: 770-8$.

46. Anttila T, Helkala EL, Viitanen M, Kareholt I, Fratiglioni L, Winblad B, et al. Alcohol drinking in middle age and subsequent risk of mild cognitive impairment and dementia in old age: a prospective population based study. BMJ. 2004;329(7465):539.

47. Stampfer MJ, Kang JH, Chen J, Cherry R, Grodstein F. Effects of moderate alcohol consumption on cognitive function in women. N Engl J Med. 2005;352(3):245-53.

48. Ujcic-Voortman JK, Schram MT, Jacobs-van der Bruggen MA, Verhoeff AP, Baan CA. Diabetes prevalence and risk factors among ethnic minorities. Eur J Public Health. 2009;19(5):511-5. 